ARTÍCULOS ORIGINALES Rev Chil Salud Pública 2017; Vol 21 (2): 142-149

\section{PERCEPCIÓN DE APOYO FAMILIAR DE ADULTOS MAYORES RESIDENTES EN ESTABLECIMIENTOS DE LARGA ESTADÍA}

Perception of Familial Support among Older Adults Living in LONG-TERM ESTABLISHMENTS

\title{
RESUMEN
}

Propósito: Describir la percepción de relaciones familiares que tienen los adultos mayores pertenecientes a Establecimientos de Larga Estadia de Temuco, 2016.

Materiales y métodos: Estudio cualitativo, fenomenológico. Se realizaron entrevistas en profundidad a diez adultos mayores residentes en diversos establecimientos de larga estadía, seleccionados por muestreo teórico. Se realizó análisis cualitativo temático utilizando NVivo11.

Resultados: Los relatos reflejan deseo de ser cuidados en sus hogares o en el de algún familiar, pero comprenden que esto es poco factible, ya que significaría una carga física, emocional y económica para el cuidador. Por tal motivo, asumen que la estadía en estos establecimientos es una buena opción. Si bien pocos entrevistados refirieron sentirse abandonados por sus familiares, todos coinciden en que existe un abandono generalizado al adulto mayor, especialmente cuando padece demencia. Los participantes sienten que, para mejorar este vínculo, solo pueden intentar evitar conflictos y mantener una buena comunicación. En todos los casos, la familia fue un factor influyente en el ingreso a la institución.

Conclusiones: El bienestar del adulto mayor institucionalizado refleja estar relacionado con el apoyo de familiares y una buena relación con ellos. El apoyo familiar mejora la calidad de vida y favorece la adaptación al lugar de residencia. Estos resultados son de importancia para formular intervenciones con un enfoque biopsicosocial. Se sugiere desarrollar y evaluar intervenciones

Lisette Gempp Escuela de Salud Pública, Universidad Mayor, Santiago, Chile

Dafna Benadof Facultad de Odontología, Universidad Andrés Bello, Santiago, Chile dafna.benadof@unab.cl que otorguen más apoyo a las familias, para que éstas realicen el cuidado del adulto mayor, sin que ello altere la dinámica familiar y calidad de vida de sus integrantes.

Palabras clave: Adulto mayor, residencia de adulto mayor, familia.

Fuente de apoyo financiero: ninguno. Trabajo autofinanciado 


\section{ABSTRACT}

Objective: To describe the perception of familial relationships of older adults living in long termestablishments in Temuco, in 2016.

Methods: Qualitative phenomenological study. We conducted 10 in depth interviews with older adults living in different long-term establishments, using a theoretical sampling process. Thematic qualitative analysis was conducted using NVivo 11.

Results: Participants' narratives reflect their wish to be taken care of at home or at a family member's home, though they understand that this is not very feasible, because it would represent a physical, emotional and financial burden for their caregiver. Therefore, they accept that being institutionalized is a good option. Even though only a few participants shared feeling abandoned by their families, all of them agree that there is a generalized abandonment of the elderly, especially if he or she has dementia. Participants feel that the only thing they can do to improve this relationship is to avoid conflicts and maintain good communication with their family members. In all cases, family was an important factor in the institutionalization of the older adults.

Conclusions: The wellbeing of institutionalized older adults appears to be related to the support of family members and having a good relationship with them. Family support improves quality of life and favors the adaptation process to the institution. These results are important for the development of interventions that use a biopsychosocial perspective. It is suggested that intervention that provide more support to families, so they are able to care for older adults without disrupting family dynamics and quality of life, should be developed and evaluated.

Keywords: Older adults, residences for older adults, family.

\section{INTRODUCCIÓN}

En Chile, entre los años 1990 y 2013, la población adulto mayor creció $121 \%$, lo que impactó considerablemente el índice de envejecimiento. ${ }^{1}$ El cuarto lugar en este índice lo ocupa la región de La Araucanía. ${ }^{2,3}$ Ante el aumento de este grupo poblacional, requirente de cuidados especiales debido a enfermedades discapacitantes, ${ }^{4,5}$ se instauraron los Establecimientos de Larga Estadía (ELEAM). Estos establecimientos otorgan cuidados a personas de 60 años o más, enfocándose en prevenir y mantener la salud, estimular la funcionalidad y reforzar las capacidades remanentes de los residentes. ${ }^{6,7}$

Estos hogares presentan una alta demanda y su ingreso no es voluntario ni espontáneo: aproximadamente un $40 \%$ llega por enfermedad, $30 \%$ por soledad o viudez, $10 \%$ por decisión familiar y $20 \%$ por falta de cuidador; otros motivos son mala relación con familiares, salud desfavorable y poco acceso a atención de especialistas. En estas instituciones un 9,5\% recibe visitas frecuentes; el resto, poco frecuentes o nunca. Se ha evidenciado menos visitas a quienes tienen un mayor grado de dependencia. $^{\text {? }}$

Se cree que los ELEAM dan solución a necesidades básicas y poseen una infraestructura adecuada, pero generan un elevado gasto económico para el país; ${ }^{8}$ al contrario, el cuidado por parte de familiares es menos costoso para el Estado y mejora la calidad de vida del adulto mayor. Por estos motivos, se postula que los adultos mayores debiesen llegar a estos establecimientos solo en casos extremos. ${ }^{9}$

El presente estudio tiene por objetivo describir cómo perciben la relación con su familia los adultos mayores residentes en ELEAM. Se espera con ello iniciar el conocimiento de un área poco evidenciada en la actualidad; y si bien existen escasas investigaciones en población adulto mayor de Temuco, ninguna persigue el objetivo antes detallado.

\section{MATERIAL Y MÉTODO}

Estudio cualitativo, fenomenológico. Se centró en las experiencias individuales de 
adultos mayores residentes tiempo completo en ELEAM de Temuco, Chile. Esta investigación fue aprobada por el Comité Ético Científico de la Universidad Mayor.

El reclutamiento de los participantes comenzó con la selección de los 10 ELEAM de Temuco con el mayor número de residentes autovalentes según los registros del SENAMA. Posteriormente, con ayuda de los directores de cada establecimiento, se indagó en el acceso a los ELEAM y el número de posibles participantes de cada uno de ellos, seleccionando, finalmente, a tres ELEAM que hicieran más fácil alcanzar los criterios muestrales definidos.

Se determinó utilizar una muestra mínima de 10 personas o hasta obtener saturación de información. Se buscó heterogeneidad de participantes sobre la base de criterios de edad, género, número de hijos, motivo de ingreso y autovalencia; se consideró como criterio de exclusión presentar demencia, depresión o dificultad para expresarse verbalmente.

La recolección de información se realizó con entrevistas en profundidad. Para ello, se elaboró una guía de preguntas, abarcando temas que dieran cumplimiento al objetivo de la investigación. Luego se realizó una entrevista piloto, modificando tres preguntas que no fueron comprendidas en su totalidad; luego se aplicó una segunda prueba, sin requerimiento de modificaciones.

Las entrevistas se realizaron en un lugar privado y con la presencia exclusiva de entrevistador y entrevistado. Previo a cada entrevista se leyó y explicó un consentimiento informado que debió ser firmado por cada participante. Todas las entrevistas fueron grabadas en formato digital.

Las entrevistas fueron transcritas y leídas por dos investigadoras, quienes calibraron el proceso de codificación con la finalidad de mantener la validez y rigurosidad. Se aplicó un análisis cualitativo temático, sistemático y secuencial. Cada tres entrevistas, se evaluó y adecuó la guía según necesidades detectadas y resultados preliminares del análisis (Figura 1). Para el análisis se utilizó software NVivo11.

Figura 1. Secuencia análisis de datos.

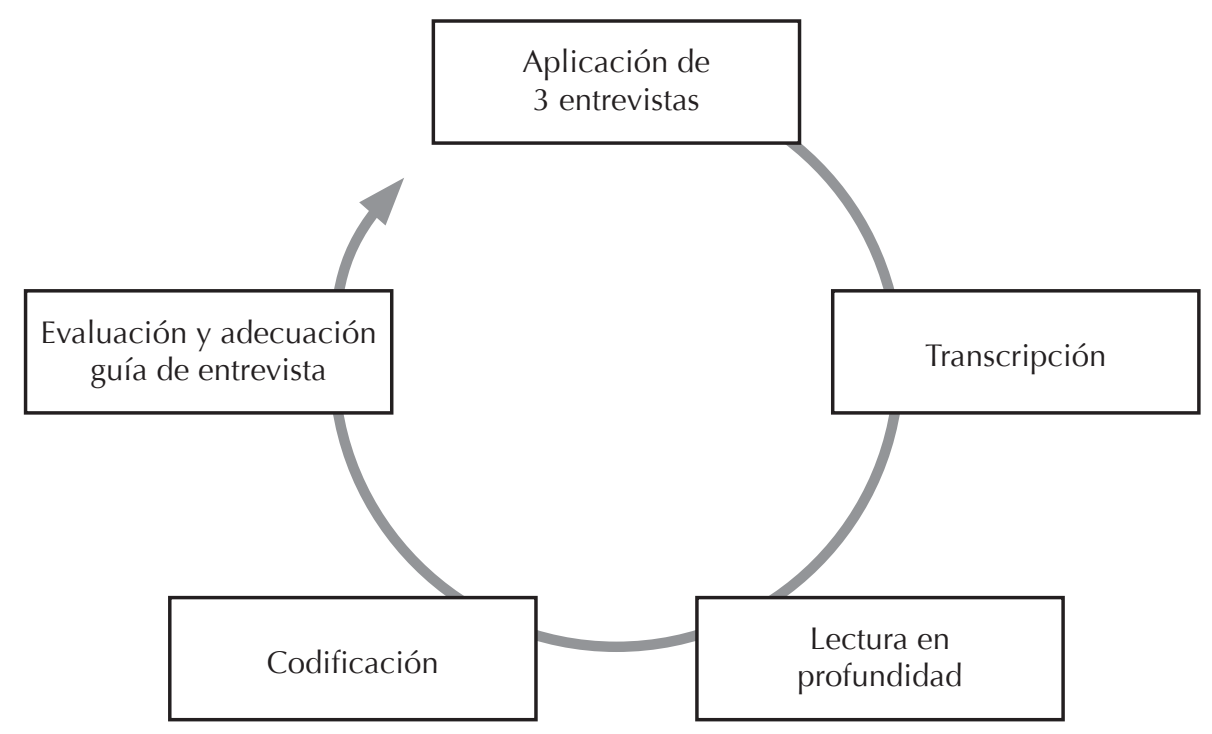




\section{RESULTADOS}

Se entrevistó a 10 sujetos entre 68 y 93 años de edad con distintos grados de dependencia, número de hijos y tiempos de estadía (Tabla 1$)$.
En relación con sus familias, los relatos de los participantes reflejaron los temas tratados a continuación.

Tabla 1. Perfil de los entrevistados

\begin{tabular}{|c|c|c|c|c|c|c|}
\hline Participante & Género & Edad & $\begin{array}{l}\text { Tiempo de } \\
\text { estadía }\end{array}$ & $\begin{array}{l}\text { Motivo de } \\
\text { ingreso }\end{array}$ & Dependencia referida & $\begin{array}{l}\text { Hijos/hijas } \\
\text { vivos }\end{array}$ \\
\hline $\mathrm{N}^{\circ} 1$ & M & 79 & 9 años & Soledad & Autovalente & Sí \\
\hline $\mathrm{N}^{\circ} 2$ & $\mathrm{~F}$ & 70 & 2 años & Soledad & Dependencia leve & Sí \\
\hline $\mathrm{N}^{\circ} 3$ & M & 68 & 2 años & Soledad & Dependencia leve & No \\
\hline $\mathrm{N}^{\circ} 4$ & M & 73 & 1 año & Enfermedad & Autovalente & Sí \\
\hline $\mathrm{N}^{\circ} 5$ & $\mathrm{~F}$ & 75 & 4 años & Edad & Dependencia leve & No \\
\hline $\mathrm{N}^{\circ} 6$ & $\mathrm{~F}$ & 87 & 2 años & $\begin{array}{l}\text { Conflictos } \\
\text { familiares }\end{array}$ & Dependencia leve & Sí \\
\hline$N^{\circ} 7$ & $\mathrm{~F}$ & 83 & 2 años & Enfermedad & Autovalente & Sí \\
\hline $\mathrm{N}^{\circ} 8$ & M & 75 & 3 meses & Enfermedad & Dependencia leve & Sí \\
\hline $\mathrm{N}^{\circ} 9$ & M & 93 & 5 meses & Soledad & Dependencia leve & Sí \\
\hline$N^{\circ} 10$ & $\mathrm{~F}$ & 86 & 10 meses & Enfermedad & Dependencia severa & No \\
\hline
\end{tabular}

\section{Motivos de ingreso al ELEAM}

Todos los entrevistados refirieron tener familia fuera del ELEAM. Los motivos de ingreso se relacionaban con: temor a envejecer y sobrecargar a la familia: "Yo pensé que iba a llegar a ser un estorbo $y$, antes de llegar a eso, preferi venirme"; padecer enfermedad que requiere cuidados especiales: "Mi bija me trajo a la casa de reposo para hacer ejercicio con la señorita kinesióloga"; tener mala relación con algún familiar: "Mi bijo yo creo que habría deseado que nos fuéramos con él, pero su esposa no"; reducir gastos económicos: “... ellos mismos empezaron a ver que era mucha la plata que estaban gastando, cuando por esa plata me podian llevar a un lugar donde me atendieran mejor"; sensación de soledad por la muerte de un familiar cercano: "Quedé dando vueltas; estuve en Arica con una hermana, murió mi hermana, me vine para acá"; y mayor dependencia física: “...después de eso nosotros nos teníamos que atender cojos y jodidos sin poder tener agilidad para eso".

Otros participantes fueron llevados por sus hijos(as), lo que percibieron como abandono: "Ingresé porque ellos buscaron un lugar para deshacerse, entre comillas, de mí, es lo que yo pienso". Un participante incluso extrapola esta impresión a todos los que llegan a un ELEAM: "A estos abuelitos y abuelitas las traen aqui, pero yo le voy a decir con toda sinceridad que los vienen a botar aqui nomás".

\section{Expectativas de apoyo familiar}

La mayoría de los entrevistados temen padecer alguna enfermedad que signifique alta demanda para sus familias: "Voy a llegar a estar más viejito, ya no voy a poder andar como ando ahora, ya después voy a empezar a tener alzhéimer, va a fallarme la memoria y después 
me voy a hacer de todo y voy a ser para ustedes una carga y eso no quiero yo". Justifican su estadía en el ELEAM, a pesar de que preferirían vivir en sus casas: "Me habría gustado que me recibiera en su casa".

Predomina una resignación que motiva el no responsabilizar a los familiares por el cuidado: "Si usted en su misma casa suya usted se casa, tiene sus hijos y tiene a su papá al lado que se le enferma, que tiene que andar para allá y para acá, es un estorbo, es algo que para usted es traumático, entonces prefieren mejor convencer al hombrecito o a la mujercita y pagar aquí para tenerlos acá".

Cuando el ingreso al ELEAM generó conflicto con familiares, prevalece el disgusto; un entrevistado expresó que aún lamenta la decisión tomada por su hija: "Le quiero tirar las orejas a mi hija que me trajo aqui un dia; nada que ver".

A pesar de lo anterior, esperan más apoyo cuando está la sensación de haber hecho mucho por el núcleo familiar, pretendiendo una cierta retribución emocional: "Yo di mi vida por ellos en un momento, entonces me duele mucho lo que hacen de estar tan desapegados".

Todos coinciden en que la visita de familiares es clave para mantener la relación cercana y sentirse parte de la familia, fomentando la calidad de vida del adulto mayor: "Obviamente que les hace súper bien; los que están más lúcidos, porque hay algunos que simplemente no cachan ni una cosa". Al respecto, algunos consideran que la demencia es un factor clave en el abandono: "Eso es lo principal, porque no están bien de la cabeza”, e intentan justificar este fenómeno, reflejando bajas expectativas de apoyo familiar: "Yo creo que eso tiene que influir porque, se me hace la idea así como que si está perdido no se va a dar ni cuenta, no nos va a echar de menos así que a qué vamos a ir".

\section{Cambio en la relación con familiares}

Hospitalizaciones prolongadas y dependencia física favorecen cambios en la relación con la familia: "Yo era como parte de la familia, o sea estaba súper integrado y habia una muy buena relación, hasta que me enfermé".
La hospitalización genera diversas reacciones por parte del adulto mayor: "Yo decía jadónde me trajo mi bija! [...] pero nunca babia ido a un hogar, nunca... entonces yo dije no... iy me largué a llorar!". En el caso de dependencia física, el constante cuidado que debe otorgar la familia y el desgaste derivado altera la dinámica normal del núcleo y aumenta los conflictos: "Mi nuera, por ejemplo, empezó a ser indiferente, me ignoraba. Esas cosas evan, pero no eran groserías ni malos tratos, no, pero la indiferencia me dolió".

Una razón del deterioro del vínculo familiar se asocia con la administración de los recursos del adulto mayor; ejemplo de ello es el relato de una entrevistada respecto a la herencia que había dejado su esposo: “...sacaron toda la plata de la cuenta RUT de mi marido y me dejaron sin nada".

\section{ELEAM, apoyo familiar y salud}

Todos los entrevistados coinciden en que la estadía en un ELEAM es favorable para la salud física por los buenos cuidados otorgados: "Me siento cómoda aqui, totalmente, yo sé que al otro día voy a amanecer viva”; "... acá [ante] cualquier cosa está la enfermería”. Sin embargo, los buenos cuidados apuntan a acciones programadas (administración de medicamentos, aseo y confort); cuando se trata de necesidades básicas (baño o cubrirse con una manta), no existe total conformidad: "Sí, me cuidan, pero yo puedo estar aqui que ya no aguanto más, no aguanto más y llamo y llamo por el famoso timbre y no viene nadie"; "pueden tenerlos destapados, con frío, sin calefacción y abi están”. Adicionalmente, los participantes reportan apoyo emocional distinto al que ellos esperarían en esta etapa de sus vidas: "Esa cosa que se llama familia, que se bace una sola, que viven los mismos problemas, que viven las mismas alegrias [...] a mi me gusta que me digan que me quieren, en fin, qué sé yo, aunque sea mentira, que no lo sientan pero que de vez en cuando me den un abrazo".

En cuanto a la relación con familiares, la disminución del contacto físico trae consecuencias anímicas significativas: “...pero el 
becho de estar aquí, el hecho ya de estar aquí ya no es una felicidad, no se lo recomiendo a nadie"; "te das cuenta cómo te vas a pique, tú misma vas mirando y viendo tu pendiente hacia abajo, y eso es nefasto".

\section{Mantención del vínculo}

La mayoría de los entrevistados se esfuerza por mantener la relación mediante visitas y llamadas telefónicas, evitando todo tipo de conflictos, como manifiestan los siguientes entrevistados: "teléfono, email, vienen, voy, señora Patricia me lleva... el domingo fui a almorzar donde mi mamá de sorpresa"; "conversamos de todo, cuando me vienen a ver 'cómo te sientes, qué has hecho', compartimos el diario vivir; me llaman y yo también los llamo a ellos".

Otros no sienten la necesidad de actuar al respecto; se trata de aquellos que perciben una buena relación con sus familias o que consideran imposible mantener una relación cercana desde el ELEAM: "No les pido nada ni las llamo, llegan solitas y me vienen a ver".

En todos los casos existe la necesidad de tener a sus familiares físicamente cerca.

\section{Sensación de abandono}

De los 10 entrevistados, dos refirieron sentirse abandonados por sus familiares. Sin embargo, todos coinciden en que existe abandono al adulto mayor en los ELEAM: "Hay cuántos que no saben, no tienen idea de cuándo han muerto, que bemos tenido que ir a dejarlos nosotros mismos porque no llega ningún familiar a la misa; no todos tampoco, pero alguno que otro que están aqui abandonados, como en todos los hogares de ancianos de Chile"; "estos tres días que se tomaron sándwich, que dicen que se toman... ino llegó nadie al hogar! Diría que tres personas de 140, para el lado de las mujeres, no sé para el lado de los hombres. No se preocupan de los mayores. Están solos... solos, solos, solos".

Intentan explicar esta situación con el estilo de vida de la población más joven, argumentando que muchos no van de visita por falta de tiempo libre; otros refieren que sería por el carácter del adulto mayor: "Para mí que el mundo no acompaña, el medio ambiente, la sociedad en que vivimos no ayuda a que tú tengas presente en tu mente $y$ en tu quehacer diario hacer una visita a un viejo o vieja que está guardado aqui"; "la vida será, digo yo, tan agitada que no tienen con quién dejar en la casa a su mamá o 'ya, dejé a esta vieja odiosa aqui', porque todo cristiano tiene sus mañas".

\section{Discusión}

Los adultos mayores expresan vivir en una constante resignación respecto a su etapa de vida; las consecuencias del paso de los años comienzan a aparecer ${ }^{10}$ y no existe gente cercana dispuesta a cuidarlos, así que ingresan al ELEAM a pesar de los sentimientos que esto pueda generar.

Varios autores coinciden en que la familia influye altamente en la institucionalización del adulto mayor ${ }^{7,11,12}$ y que el apoyo emocional mejora la calidad de vida y favorece la adaptación de éstos. ${ }^{12}$ Se sabe que el cuidado de un adulto mayor genera una alta sobrecarga. ${ }^{13,14}$ Sin embargo, parece lógico esperar que la familia no se desligue completamente del cuidado. ${ }^{8,9}$ En algunos casos, junto con abandonar esta responsabilidad, además existe apropiación de recursos, generando sensación de angustia y pérdida de pertenencia dentro del núcleo. Varios estudios refieren que más de la mitad de los adultos mayores institucionalizados se sienten abandonados. ${ }^{7,11}$

Según los resultados aquí obtenidos, la estadía en un ELEAM de por sí afecta el estado anímico, aumentando el desánimo con el desinterés de familiares; estudios cuantitativos han mostrado que cerca del $50 \%$ de los residentes en asilos de ancianos sufre síntomas depresivos; ${ }^{15,16}$ misma cantidad para quienes creen que la institucionalización no satisface sus necesidades básicas. ${ }^{17}$ Estos datos invitan a reflexionar sobre la necesidad de apoyo familiar a este grupo de la población.

Los ELEAM, según los resultados de este estudio, proveen cuidados óptimos y promueven la salud física, lo que coincide con otras investigaciones. $^{12,18}$ Bajo esta premisa, un 
ELEAM podría considerarse un apoyo importante a los establecimientos de salud que, habitualmente, son muy demandados por personas mayores. ${ }^{19}$

Los resultados obtenidos ayudan a prever el futuro: Chile envejecido, con adultos mayores viviendo fuera de sus hogares, afectados emocionalmente, con aumento exponencial de casas de reposo y familiares desgastados por el cuidado de quienes alguna vez los cuidaron a ellos. Según Vergara, ${ }^{20}$ el insuficiente conocimiento científico que existe hasta ahora respecto del adulto mayor institucionalizado ha dificultado la implementación de estrategias que mejoren su calidad de vida en Chile. Si bien existen medidas ya implementadas, como el Programa del Adulto Mayor para prevención, tratamiento y rehabilitación de enfermedades, Programa de Envejecimiento Activo, patologías GES, atención domiciliaria a personas postradas (adultos mayores en su mayoría) con apoyo de diverso tipo a los cuidadores, ELEAM diurnos y de residencia completa, Programa de Cuidados Domiciliarios, entre otros, no todas esas medidas otorgan bienestar emocional.

Esta investigación es un aporte significativo a la Política Nacional para el Adulto Mayor, la cual tiene por objetivo "Lograr un cambio cultural de toda la población sobre la valoración y trato hacia los Adultos Mayores, lo cual implica una percepción distinta sobre el envejecimiento y, alcanzar mejores niveles de calidad de vida para todos los Adultos Mayores". 8

No solo en Chile existe preocupación por la salud del adulto mayor. Así, por ejemplo, la Secretaría General Iberoamericana (SEGIB) y la Organización Iberoamericana de Seguridad Social (OISS) han propuesto un Programa Iberoamericano de los Adultos Mayores, para conocer y mejorar la situación de este grupo de la población en países como Argentina, Brasil, Chile, Ecuador, España, México, Paraguay, Uruguay. Una de las estrategias a las que invita este programa, es a fomentar la investigación cualitativa sobre la situación de los adultos mayores en cada país.

Este estudio ha permitido conocer una realidad de la que existe poca evidencia a nivel nacional. Si bien no es posible generalizar los resultados aquí obtenidos, se ha generado un fundamento científico para nuevos estudios que busquen el bienestar del adulto mayor, y una alarma que permite generar conciencia respecto a lo mismo; ya no es un mito popular que los adultos mayores se sienten abandonados; ahora existe un estudio que confirma que ellos requieren más apoyo de sus familiares. Merecen ser cuidados con amor, respeto $\mathrm{y}$ dignidad.

Una buena forma de trabajar hacia ese punto es aumentar el foco de la investigación hacia la prevención de patologías psíquicas que generan dependencia en el adulto mayor. Junto con ello, masificar los ELEAM de residencia diurna y la cobertura del Programa de Cuidados Domiciliarios, de modo que los familiares no entreguen toda la responsabilidad al Estado que, por cierto, es probable que no logre garantizar el cuidado de una población tan masiva; por supuesto, evaluando previamente el impacto económico y en salud. Asimismo, la familia sin duda requiere de apoyo que facilite la tarea del cuidado, para que no altere la dinámica familiar ni la calidad de vida de sus integrantes.

Sería interesante investigar cómo viven el proceso de envejecimiento los adultos mayores del pueblo mapuche, población numerosa en el sur de Chile, para implementar medidas de salud pública adaptadas a la necesidad de cada cultura.

\section{CONCLUSIÓN}

El bienestar del adulto mayor institucionalizado refleja estar relacionado con el apoyo de familiares y una buena relación con ellos. El apoyo familiar mejora la calidad de vida y favorece la adaptación al lugar de residencia. Estos resultados son de importancia para formular intervenciones con un enfoque biopsicosocial. Se sugiere evaluar intervenciones que otorguen más apoyo a las familias, para que éstas realicen el cuidado del adulto mayor, sin que esto altere la dinámica familiar y calidad de vida de sus integrantes. 


\section{REFERENCIAS}

1. Chile. Ministerio de Desarrollo Social. Informe de desarrollo social 2015. Santiago: Ministerio de Desarrollo Social; 2015.

2. Instituto Nacional de Estadísticas (Chile). Compendio estadístico regional La Araucanía. Temuco: INE; 2015.

3. Chile. Ministerio de Desarrollo Social. Casen 2013, adultos mayores. Santiago: Ministerio de Desarrollo Social; 2013.

4. Olivaries P. Perfil Epidemiológico del Adulto Mayor en Chile. Santiago: Departamento de Estudios y Desarrollo; 2006.

5. Ibáñez C, España M, Miranda V, Gillmore M. Dependencia de los Adultos Mayores en Chile. Santiago: Departamento de Estudios y Desarrollo; 2008.

6. Chile. Ministerio de Salud. Departamento de Asesoría Jurídica. Reglamento de Establecimientos de Larga Estadía para Adultos Mayores. 2005

7. Universidad Academia de Humanismo Cristiano (Chile). Diagnóstico de la situación de los adultos mayores residentes en Establecimientos de Larga Estadía en regiones, informe final. Santiago: Universidad Academia de Humanismo Cristiano; 2007.

8. Servicio Nacional del Adulto Mayor (Chile). Política nacional para el adulto mayor. Santiago: SENAMA; 2004.

9. Servicio Nacional del Adulto Mayor (Chile). Politica integral de envejecimiento positivo para Chile. Santiago: SENAMA; 2012.

10. Durán D, Orbegoz L, Uribe A, Uribe J. Integración social y habilidades funcionales en adultos mayores. Univ Psychol. 2008;7(1):263-70. Disponible en: www.redalyc.org/articulo.oa?id=64770119 [consultado el 10.05.2016].

11. Cardona D, Estrada A, Chavarriaga L, Segura Á, Ordóñez J, Osorio J. Apoyo social dignificante del adulto mayor institucionalizado. Rev Salud Pública. 2010; 12(3): 414-24.
12. Contreras J, Suárez A, Comelin A. Efectos psicosociales de la desvinculación familiar desde la percepción de los adultos mayores autovalentes en situación de abandono familiar, en el Establecimiento de Larga Estadía del Adulto Mayor "Huerto de Paz" de la comuna de Alto Hospicio [tesis]. Iquique: Universidad Arturo Prat; 2014.

13. Cardona D, Segura A, Berbesí D, Agudelo M. Prevalencia y factores asociados al síndrome de sobrecarga del cuidador primario de ancianos. Rev Fac Nac Salud Pública. 2013; 31(1): 30-9.

14. Valle M, Hernández I, Zúñiga M, Martínez P. Sobrecarga y burnout en cuidadores informales del adulto mayor. Enfermería Univ. 2015; 12(1): 19-27.

15. Estrada A, Cardona D. Síntomas depresivos en adultos mayores institucionalizados y factores asociados. Univ Psychol. 2013; 12(1): 81-94.

16. Borda M, Anaya M, Milena M, Romero L, Suárez A, Suárez A. Depresión en adultos mayores de cuatro hogares geriátricos de Barranquilla: prevalencia y factores de riesgo. Salud Uninorte. 2013; 29(1): 64-73.

17. Sepúlveda C, Rivas E, Bustos L, Illesca M. Perfil socio-familiar en adultos mayores institucionalizados: Temuco, Padre Las Casas y Nueva Imperial. Cienc enferm. 2010; 16(3): 49-61.

18. Santa Cruz M. Proceso de adaptación de los adultos mayores al ingreso en el asilo de ancianos [tesis]. Chiclayo: Universidad Católica; 2013.

19. Universidad Técnica Federico Santa María. Demanda de servicios de cuidado para las personas mayores. Valparaíso: Universidad Técnica Federico Santa María; 2009.

20. Vergara S. Residencias para adultos mayores: criterios básicos para su adecuada selección. Rev Hosp Clín Univ Chile. 2008; 19 : 356-60. 